\title{
Degrees of Non-computability of Homeomorphism Types of Polish Spaces
}

\author{
Mathieu Hoyrup ${ }^{1}$, Takayuki Kihara ${ }^{2(\bowtie)}$, and Victor Selivanov ${ }^{3,4}$ \\ 1 Université de Lorraine, CNRS, Inria, LORIA, 54000 Nancy, France \\ mathieu.hoyrup@inria.fr \\ 2 Nagoya University, Nagoya 464-8601, Japan \\ kihara@i.nagoya-u.ac.jp \\ 3 A.P. Ershov Institute of Informatics Systems SB RAS, Novosibirsk, Russia \\ vseliv@iis.nsk.su \\ ${ }^{4}$ Kazan Federal University, Kazan, Russia
}

\begin{abstract}
There are continuum many homeomorphism types of Polish spaces. In particular, there is a Polish space which is not homeomorphic to any computably presented Polish space. We examine the details of degrees of non-computability of presenting homeomorphic copies of Polish spaces.
\end{abstract}

Keywords: Computable topology $\cdot$ Computable presentation • Computable Polish space $\cdot$ Degree spectrum

How difficult is it to describe an explicit presentation of an abstract mathematical structure? In computable structure theory, there are a large number of works on degrees of non-computability of presenting isomorphism types (known as degree spectra) of algebraic structures such as groups, rings, fields, linear orders, lattices, Boolean algebras, and so on, cf. $[1,5,6,8]$. In this article, we deal with its topological analogue, i.e., degrees of non-computability of presenting homeomorphism types of certain topological spaces, and mainly focus on presentations of Polish spaces (i.e., completely metrizable separable spaces). We present some of the results obtained in Hoyrup-Kihara-Selivanov [9] on this topic.

The notion of a presentation plays a central role, not only in computable structure theory, but also in computable analysis $[2,3,16]$. In this area, one of the most crucial problems was how to present large mathematical objects (which possibly have the cardinality of the continuum) such as metric spaces, topological spaces and so on, and then researchers have obtained a number of reasonable answers to this question. In particular, the notion of a computable presentation of

The second-named author was partially supported by JSPS KAKENHI Grant 19K03602, 15H03634, and the JSPS Core-to-Core Program (A. Advanced Research Networks). The third-named author was supported by the Russian Science Foundation project No 18-11-00028.

(C) Springer Nature Switzerland AG 2020

M. Anselmo et al. (Eds.): CiE 2020, LNCS 12098, pp. 189-192, 2020.

https://doi.org/10.1007/978-3-030-51466-2_16 
a Polish space has been introduced around 1950-60s, cf. [12], and since then this notion has been widely studied in several areas including computable analysis $[2,14,16]$ and descriptive set theory [13].

In recent years, several researchers succeeded to obtain various results on Turing degrees of presentations of isometric isomorphism types of Polish metric spaces, separable Banach spaces, and so on, cf. [4,10,11]. However, most of works are devoted to metric structures, and there seem almost no works on presentations on homeomorphism types of Polish spaces. The investigation of Turing degrees of homeomorphism types of topological spaces (not necessarily Polish) was initiated in [15]. Some results were obtained for domains but the case of Polish spaces was apparently not investigated seriously so far.

Every Polish space is homeomorphic to the Cauchy completion of a metric on (an initial segment of) the natural numbers $\omega$, so one may consider any distance function $d: \omega^{2} \rightarrow \mathbb{Q}$ as a presentation of a Polish space. Then, observe that there are continuum many homeomorphism types of Polish spaces. In particular, by cardinality argument, there is a Polish space which is not homeomorphic to any computably presented Polish space. Surprisingly however, it was unanswered until very recently even whether the following holds:

Question 1. Does there exist a $0^{\prime}$-computably presented Polish space which is not homeomorphic to a computably presented one?

The solution to Question 1 was very recently obtained by the authors of this article, and independently by Harrison-Trainor, Melnikov, and Ng [7]. One possible approach to solve this problem is using Stone duality between countable Boolean algebras and zero-dimensional compact metrizable spaces, but they also

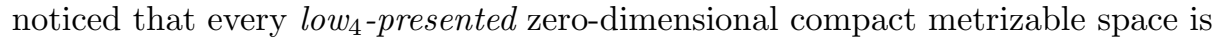
homeomorphic to a computable one. This reveals certain limitations of Stone duality techniques. Our next step is to develop new techniques other than Stone duality. More explicitly, the next question is the following:

Question 2. Does there exist a low -presented Polish space which is not homeomorphic to a computably presented one?

One of the main results in Hoyrup-Kihara-Selivanov [9] is that there exists a $0^{\prime}$-computable $l o w_{3}$ infinite dimensional compact metrizable space which is not homeomorphic to a computable one. This solves Question 2. Indeed, HoyrupKihara-Selivanov [9] showed more general results by considering two types of presentations:

A Polish presentation (or simply a presentation) of a Polish space $\mathcal{X}$ is a distance function $d$ on $\omega$ whose Cauchy completion is homeomorphic to $\mathcal{X}$. If $\mathcal{X}$ is moreover compact, then by a compact presentation of $\mathcal{X}$ we mean a presentation of $\mathcal{X}$ equipped with an enumeration of (codes of) all finite rational open covers of $\mathcal{X}$, where a rational open set is a finite union of rational open balls. For readers who are familiar with the abstract theory of modern computable analysis (cf. [2]), we note that a Polish (compact, resp.) presentation of $\mathcal{X}$ is just a name of a homeomorphic copy of $\mathcal{X}$ in the space of overt (compact overt, resp.) subsets of Hilbert cube $[0,1]^{\omega}$. 
Theorem 1 ([9]). For any Turing degree $\mathbf{d}$ and natural number $n>0$, there exists a compact metrizable space $\mathcal{Z}_{\mathbf{d}, n}$ such that for any Turing degree $\mathbf{x}$,

$$
\mathbf{d} \leq \mathbf{x}^{(2 n-1)} \Longleftrightarrow \mathcal{Z}_{\mathbf{d}, n} \text { has an } \mathbf{x} \text {-computable compact presentation. }
$$

Theorem 2 ([9]). For any Turing degree $\mathbf{d}$ and natural number $n>0$, there exists a compact metrizable space $\mathcal{P}_{\mathbf{d}, n}$ such that for any Turing degree $\mathbf{x}$,

$$
\begin{aligned}
\mathbf{d} \leq \mathbf{x}^{(2 n)} & \Longleftrightarrow \mathcal{P}_{\mathbf{d}, n} \text { has an } \mathbf{x} \text {-computable compact presentation } . \\
\mathbf{d} \leq \mathbf{x}^{(2 n+1)} & \Longleftrightarrow \mathcal{P}_{\mathbf{d}, n} \text { has an } \mathbf{x} \text {-computable Polish presentation } .
\end{aligned}
$$

Corollary 1 ([9]). There exists a Polish space which is $\mathbf{0}^{\prime}$-computably presentable, but not computably presentable.

Indeed, for any $n>0$, there exists a compact metrizable space $\mathcal{X}$ such that

$$
\begin{aligned}
\mathbf{x} \text { is high } & \Longleftrightarrow \mathcal{X} \text { has an } \mathbf{x} \text {-computable compact presentation } . \\
\mathbf{x} \text { is high } & \Longleftrightarrow \mathcal{X} \text { has an } \mathbf{x} \text {-computable Polish presentation. }
\end{aligned}
$$

Corollary 2 ([9]). There is a compact Polish space $\mathcal{X}$ which has a computable presentation, but has no presentation which makes $\mathcal{X}$ computably compact.

Another important question is whether the homeomorphism type of a Polish space can have an easiest presentation.

Question 3. Does there exist a homeomorphism type of a Polish space which is not computably presentable, but have an easiest presentation with respect to Turing reducibility?

Hoyrup-Kihara-Selivanov [9] partially answered Question 3 in negative. More precisely, we show the cone-avoidance theorem for compact Polish spaces, which states that, for any non-c.e. set $A \subseteq \omega$, every compact Polish space has a presentation that does not enumerate $A$.

Some other side results in [9] are:

Theorem 3 ([9]). There exists a compact metrizable space $\mathcal{X}$ which has a computably compact presentation, but its nth Cantor-Bendixson derivative $\mathcal{X}^{n}$ has no $\mathbf{0}^{(2 n-1)}$-computably compact presentation.

Indeed, there exists a compact metrizable space $\mathcal{X}$ such that the following are equivalent for any Turing degree $\mathbf{x}$ :

1. $\mathcal{X}$ has an $\mathbf{x}$-computable compact presentation

2. The $n$th derivative $\mathcal{X}^{n}$ has an $\mathbf{x}^{(2 n)}$-computable compact presentation

Theorem 4 ([9]). There exists a computably presentable compact metrizable space whose perfect kernel is not computably presentable.

Similarly, there exists a computably compact, computably presented, compact metrizable space whose perfect kernel is compact, but has no presentation which makes it computably compact. 
Our key idea is using dimension (more explicitly, high-dimensional holes, i.e., a cycle which is not a boundary) to code a given Turing degree. As a result, all of our examples in the above results are infinite dimensional. We do not know if there are finite dimensional examples satisfying our main results. We also note that all of our examples are disconnected, and it is not known if there are connected examples.

\section{References}

1. Ash, C.J., Knight, J.: Computable Structures and the Hyperarithmetical Hierarchy, Studies in Logic and the Foundations of Mathematics, vol. 144. North-Holland Publishing Co., Amsterdam (2000)

2. Brattka, V., Hertling, P.: Handbook of Computability and Complexity in Analysis (202x)

3. Brattka, V., Hertling, P., Weihrauch, K.: A tutorial on computable analysis. In: Cooper, S.B., Löwe, B., Sorbi, A. (eds.) New Computational Paradigms, pp. 425491. Springer, New York (2008). https://doi.org/10.1007/978-0-387-68546-5_18

4. Clanin, J., McNicholl, T.H., Stull, D.M.: Analytic computable structure theory and $L^{p}$ spaces. Fund. Math. 244(3), 255-285 (2019)

5. Ershov, Y.L., Goncharov, S.S., Nerode, A., Remmel, J.B., Marek, V.W. (eds.): Handbook of recursive mathematics. In: vol. 2, Studies in Logic and the Foundations of Mathematics, vol. 139. North-Holland, Amsterdam (1998). Recursive algebra, analysis and combinatorics

6. Fokina, E.B., Harizanov, V., Melnikov, A.: Computable model theory. In: Turing's Legacy: Developments from Turing's Ideas in Logic. Lecture Notes in Logic, vol. 42, pp. 124-194. Association for Symbolic Logic, La Jolla (2014)

7. Harrison-Trainor, M., Melnikov, A., Ng, K.M.: Computability up to homeomorphism (2020)

8. Hirschfeldt, D.R., Khoussainov, B., Shore, R.A., Slinko, A.M.: Degree spectra and computable dimensions in algebraic structures. Ann. Pure Appl. Logic 115(1-3), 71-113 (2002)

9. Hoyrup, M., Kihara, T., Selivanov, V.: Degree spectra of Polish spaces (2020, in preparation)

10. McNicholl, T.H., Stull, D.M.: The isometry degree of a computable copy of $\ell^{p 1}$. Computability 8(2), 179-189 (2019)

11. Melnikov, A.G.: Computably isometric spaces. J. Symb. Logic 78(4), 1055-1085 (2013)

12. Moschovakis, Y.N.: Recursive metric spaces. Fund. Math. 55, 215-238 (1964)

13. Moschovakis, Y.N.: Descriptive Set Theory, Mathematical Surveys and Monographs, vol. 155, 2nd edn. American Mathematical Society, Providence (2009)

14. Pour-El, M.B., Richards, J.I.: Computability in Analysis and Physics. Perspectives in Mathematical Logic (1989)

15. Selivanov, V.: On degree spectra of topological spaces (2019)

16. Weihrauch, K.: Computable Analysis, An Introduction. Texts in Theoretical Computer Science. An EATCS Series. Springer, Berlin (2000). https://doi.org/10.1007/ 978-3-642-56999-9 